\title{
ANÁLISIS PLURIESCALAR DE LOS PATRONES DE DISTRIBUCIÓN ESPACIAL DEL ENDEMISMO ALICANTINO Vella lucentina, MB CRESPO (Brassicaceae) E IMPLICACIONES SOBRE SU CONSERVACIÓN*
}

\author{
GRAS, M.J. ${ }^{1}$, RAVENTÓS, J. ${ }^{1}$, BONET, A', RAMÍREZ, D.A. ${ }^{2}$ \\ ${ }^{1}$ Departamento de Ecología. Universidad de Alicante. Ap. 99. 03080 Alicante. \\ 2 Laboratorio de Ecología de Procesos. Universidad Nacional Agraria La Molina. \\ Ap. 456 Lima. Perú. e-mail: mj.gras@ua.es
}

\begin{abstract}
Resumen: El análisis de la distribución de los patrones espaciales y de la estructura de las poblaciones en el espacio, es uno de los aspectos clave que se deben considerar en la gestión y conservación de poblaciones de especies vegetales. En el presente estudio se realiza un análisis de la distribución del arbusto endémico estricto de la provincia de Alicante, Vella lucentina, con el fin de obtener información acerca de su biología que favorezca la gestión adecuada de las poblaciones existentes para asegurar su conservación. El estudio contempla dos escalas espaciales. En primer lugar, en la escala regional, se analiza la estructura metapoblacional de la especie dentro de la Provincia de Alicante (Comunidad Valenciana). En segundo lugar, el análisis en una escala local permite apreciar la existencia de discontinuidades en la densidad de los individuos dentro de una misma población, en función de las coberturas de suelo.
\end{abstract}

Palabras clave: corologia, endemismo, macroescala, mesoescala, fragmentación de hábitats, conservación, Vella lucentina, semiárido.

\begin{abstract}
Spatial pattern in plant populations is a key topic in conservation ecology of rare plants. We analyse distribution patterns of rare, endemic shrub Vella lucentina in semi-arid southeastern Spain at macro and mesoescale. Macroescale analysis show a metapopulation pattern whereas mesoescale analyses indicate discontinuities between plant densities related to landuses. In addition these results can offer a conceptual framework for the population management and the conservation of the species.
\end{abstract}

Keywords: Spatial pattern, scale dependency, endemic species, habitat fragmentation, conservation, Vella lucentina, semi-aricl ecosystem.

* Recibido: 3-7-2001. Aceptado: 16-7-2002. 


\section{Introducción}

El estudio de los patrones de distribución espacial de las especies de plantas raras, endémicas o amenazadas, es un aspecto fundamental de la ecología de la conservación (Fahrig y Merriam, 1994), ya que permite interpretar algunos de los aspectos más importantes de la biología de la especie y el estudio de la viabilidad de las poblaciones en áreas fragmentadas. Este tipo de análisis puede favorecer la localización de la especie, la determinación del área de distribución geográfica de sus poblaciones, el análisis de la continuidad espacial de las mismas dentro de un mismo hábitat, la densidad, la estructura en unos ambientes determinados y finalmente, distinguir la proporción de hábitats disponibles ocupados por la especie (Kunin y Gaston, 1997). Todos estos aspectos determinan, en primer lugar, la forma de rareza (Ravinowitz, 1981; Rey Benayas et al., 1999), y por otro lado, la dinámica de las poblaciones en el espacio (Brown et al., 1995), las cuales a su vez permiten estimar el grado che vulnerabilidad frente a la estocasticidad demográfica y ambiental (Lande, 1988). El conocimiento de estos elementos es esencial tanto para el desarrollo de estrategias de conservación de especies vegetales amenazadas, como para establecer criterios de manejo de las especies vegetales en hábitats heterogéneos (Gilpin, 1987; Menges, 1990; Burgman et al., 1993; Fahrig y Merriam, 1994).

Otra de las aplicaciones del estudio de los patrones espaciales es proporcionar información de cuál ha sido el efecto que las perturbaciones debidas a factores abióticos sobre la especie en un determinado hábitat, pues su distribución puede estar afectada por dicha perturbación y puede ser, eventualmente, la causa de extinciones locales (Caswell y Cohen, 1991; Wei y Skarpe, 1995). Por ejemplo, en muchas zonas áridas, y como resultado del flujo laminar en las pendientes tras las precipitaciones, se genera una estructura espacial en forma de mosaico, en el que las zonas sin vegetación se alternan con zonas de vegetación densa (Montaña, 1992; Dale, 1999).

Cabe considerar, por otra parte, el efecto de la escala en la distribución espacial de las especies vegetales, pudiendo presentar distintos patrones de distribución según el nivel analizado. A escala regional, el efecto de las perturbaciones es determinante y se pueden establecer factores selectivos en la evolución de una especie (Menaut et al. 1990). Además, el efecto de las actividades antrópicas cobra especial relevancia a esta escala, ya que pueden alterar el patrón espacial de una especie (Tongway y Ludwig, 1990), por lo que se hace especialmente importante el análisis de la distribución, considerando los diferentes usos del suelo como factor diferencial de las perturbaciones antrópicas (Naito y Nakagoshi, 1995). En concreto, en la Comunidad Valenciana, las amenazas más comunes a las que se ven sometidas las especies vegetales que han reducido sus áreas de distribución han sido: la explotación de canteras, el sobrepastoreo, la expansión urbanística y el aprovechamiento turístico masivo, la creación de nuevas redes viarias, la elevada frecuencia de incen- 
dios, la instalación de vertederos de residuos sólidos y el uso de insecticidas o pesticidas (Laguna et al., 1998).

En este trabajo se describe la distribución espacial a macro y meso-escala (sensu Dramstad et al.,1996) de la especie arbustiva Vella lucentina, M.B. Crespo. Los objetivos que abarca este estudio son: a) delimitar el área de distribución de la especie en las tres poblaciones (macro-escala), b) Realizar un estudio más detallado de la distribución y densidad de los individuos utilizando el mayor fragmento de su área de distribución (meso-escala), c) determinar el efecto que los tipos de cobertura del suelo puedan producir en los patrones de distribución espacial de la especie, y d) sobre la base de los anteriores puntos, analizar la tipología de la rareza de la especie y proponer criterios para su conservación.

\section{Metodología}

\subsection{Vella lucentina}

Vella lucentina M.B. Crespo (Brassicaceae) es un arbusto leñoso de hasta $50 \mathrm{~cm}$ de altura endémica de zonas áridas. Los tallos son glabrescentes y muy ramificados. Poseen hojas enteras lineares y estrechas e hirsutas. Las flores se disponen en racimos de 4 a 10. El fruto es una silícula dehiscente con dos valvas. Las semillas son cilíndricas de dimensiones $2 \times 1 \mathrm{~mm}$ (Crespo, 1992).

El género Vella es endémico de las áreas del suroeste Mediterráneo, específicamente en España, Marruecos y Argelia (Greuter et al. 1986). Las dos especies $V$. lucentina y $V$. spinosa encontradas en la Comunidad Valenciana presentan ciertas características morfológicas parecidas, pero se desarrollan en ámbitos altitudinales diferentes, a $350 \mathrm{~m}$ de altitud y a $1500 \mathrm{~m}$ respectivamente, aunque puede considerarse que ambas derivan de un antecesor común (Crespo, 2000). Estas a su vez se encuentran relacionadas con la especie V. pseudocytisus subs. paui (Sainz et al., 1996), localizada en las zonas áridas de Teruel y Zaragoza la cual se desarrolla entre los $960 \mathrm{~m}$ y $1200 \mathrm{~m}$ de altitud.

V. Lucentina se considera un endemismo estricto de la Provincia de Alicante catalogada como especie vulnerable (VU) según los criterios de la U.I.C.N. (1994). En general este criterio se ha aplicado a especies con un área de distribución menor a $100 \mathrm{~km}^{2}$ o de las que se conozcan menos de cinco localidades (Laguna y Crespo, 1996). Observaciones realizadas por algunos autores señalan que la vulnerabilidad de la especie se acentúa debido a que se desarrolla en suelos sometidos a factores de riesgo, como las urbanizaciones y las actividades agropecuarias (Crespo, 1992). 
Los primeros datos de la presencia de la especie Vella lucentina se proporcionaron en el año 1992, localizando el holotipo en la población del Bec de l'Aguila (30SYH1560) a $350 \mathrm{~m}$ de altitud, en el termino municipal de Muchamiel (Alicante). El área de distribución que se le atribuyó en un principio fue de $0.5 \mathrm{~km}^{2}$, posteriormente se descubrió otra población que incrementó la superficie de distribución a $2 \mathrm{~km}^{2}$ (Lledó, 1995). Actualmente se conocen 3 poblaciones localizadas en los términos municipales de Muchamiel, Jijona, Monforte del Cid y San Vicente del Raspeig (Crespo, 1996), todas ellas en condiciones ambientales semiáridas de la Provincia de Alicante (Laguna et al. 1998).

Actualmente, la conservación de esta especie se gestiona desde la Consellería de Medio Ambiente de la Generalitat Valenciana, incluyéndola en listados de flora rara, endémica o amenazada. En 1994 se creó una microrreserva (Decreto 218/1994 del Consell de la Generalitat Valenciana) para la especie Vella lucentina incluida en la Red de Microrreservas de Flora de la Comunidad Valenciana (Laguna, 1994,1995) y financiada con los fondos LIFE de la Unión Europea. Además se han realizado bancos de germoplasma (Lledó, 1995), estudios sobre la biología, estructura y dinámica de la población de la especie (Gras, 2001) y protocolos de germinación ex situ (Laguna et al. 1998).

\section{2. Área de estudio}

El estudio se ha realizado, en los términos municipales de Alicante, Jijona y Muchamiel pertenecientes a la provincia de Alicante $\left(38^{\circ} \mathrm{N}, 0^{\circ} \mathrm{W}\right)$. La altitud a la que se encuentra las poblaciones varia entre $350 \mathrm{~m}$ y $600 \mathrm{~m}$ sobre el nivel del mar.

El área de distribución de Vella lucentina, se sitúa en la parte oriental de las cordilleras Béticas, en la provincia de Alicante (Figura 1). Litológicamente, los materiales más representativos del sector prebético analizado son las calizas y margas aboudinadas, la caliza areniscosa y margas arenosas. Los materiales están expuestos a un alto grado de tectonización (IGME, 1978) y se caracterizan por presentar nódulos de hierro, que destacan dando una tonalidad ocre al paisaje.

El clima de la zona de estudio es mediterráneo, presentando dos periodos diferenciados, uno con fuertes lluvias torrenciales en la estación de otoño-invierno y otro muy seco en verano. En ocasiones se da también un periodo húmedo en primavera (Pérez-Cueva, 1994). El índice de termicidad indica que dentro del bioclima Mediterráneo xérico oceánico, esta zona pertenece al termotipo Termomediterráneo. El ombrotipo es semiárido (Rivas-Martínez, 1983). Durante los años de estudio, 1998 y 1999, la precipitación anual fue de $250 \mathrm{~mm}$ en el primer año y de $225 \mathrm{~mm}$ en 1999 (Figura 2). 


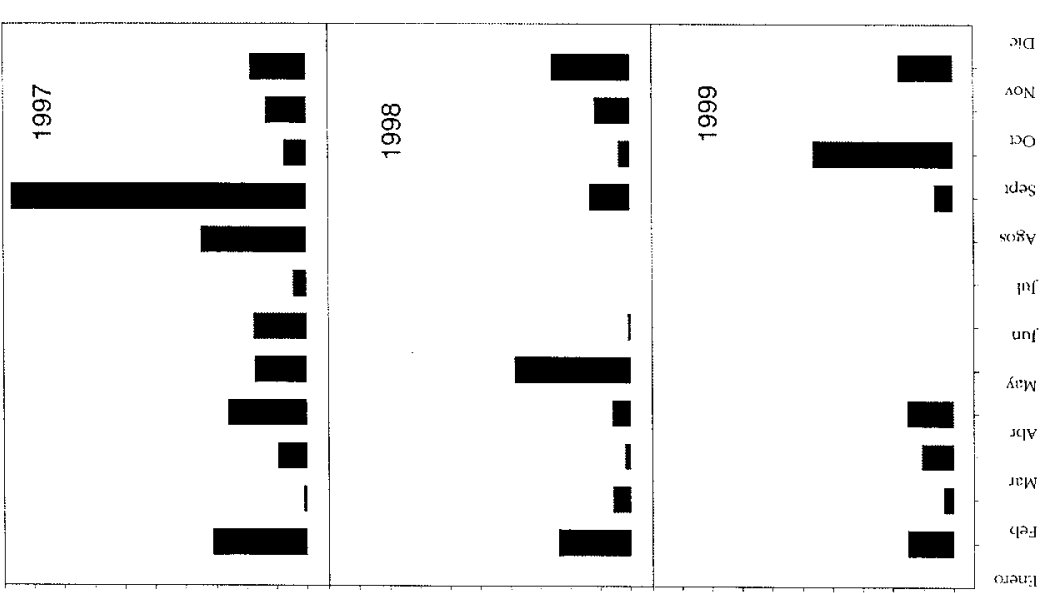

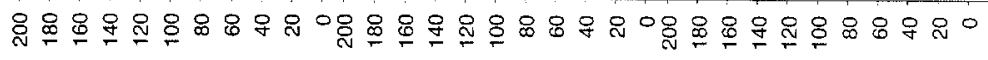

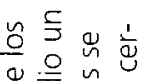

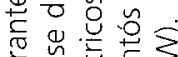

言要

응 है $\geq$

宩

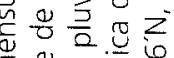

है

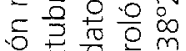

0

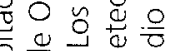

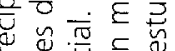

든

$\frac{8}{0} \overline{0}$

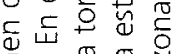

E

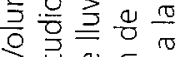
论

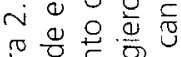

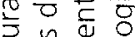

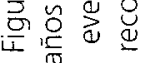

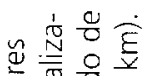

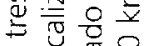

需 음 $\frac{0}{0} \frac{0}{x}$

웅

ํㅜㄹ

\%

एव

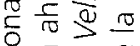

드웡

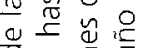

뭉

등 $\frac{\pi}{0} \cdot \frac{\xi}{0}$

웅

응 응

음

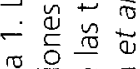

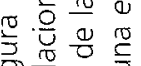

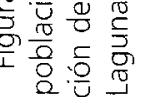


En el área de estudio se han determinado distintas coberturas de suelo, algunas de ellas muy antropizadas, como áreas de cultivos arbóreos abandonados sobre bancales (almendros, algarrobos, etc.), infraestructuras viarias (márgenes de caminos) y urbanizaciones, repoblaciones de Pinus balepensis y, más esporádicamente, de Cupressus sepervirens realizadas mediante el método de subsolado lineal. Otros sectores conservan su estado natural como las laderas ocupadas por vegetación predominantemente herbácea y subarbustiva (espartales de Stipa tenacisima), o por vegetación subarbustiva escasa sobre afloramientos rocosos (Figura 3).
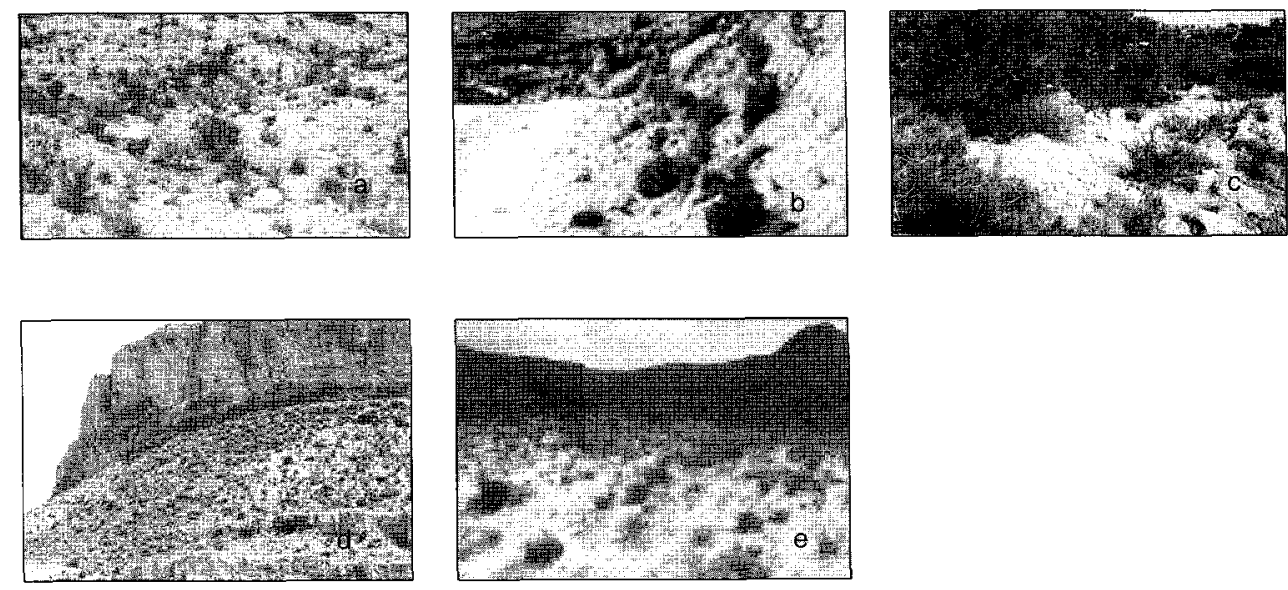

Figura 3. Coberturas de suelo en la zona de estudio: a) ladera, b) camino, c) repoblación, d) espartal, e) bancal.

\subsection{Distribución espacial a escala regional: Macro-escala}

Como primera aproximación al estudio de la distribución espacial, el análisis corológico ha empleado la base topográfica a escala 1:50000, a partir de la que se ha realizado una estimación del área de distribución ocupada por las tres poblaciones de Vella lucentina identificadas y descritas hasta el momento. En un cuadrante de coordenadas 30SHY 4805, 30SHY6205, 30SHY4819, 30SHY6219, y cubriendo el área de distribución potencial de la especie (sector semiárido de la Provincia de Alicante), se hizo un muestreo de 319 cuadriculas de $10 \times 10 \mathrm{~m}$. En cada una de ellas se tomaron datos de densidad de individuos de Vella lucentina, y los resultados obtenidos se evaluaron mediante el método de interpolación lineal Kriging (Rossi et al. 1992) para obtener una estimación en el ámbito provincial de la presencia de la especie. 


\subsection{Distribución espacial a escala local: Meso-escala}

El análisis se realizó en la población de Bec de l'Aguila (Muchamiel, Alicante, San Vicente), considerando las distintas coberturas de suelo y la densidad de individuos de distintas clases de tamaño pertenecientes a Vella lucentina. En dicha población se encuentra ubicada una microrreserva de flora, además de ser la más amplia de las tres poblaciones identificadas.

En primer lugar se determinaron los límites del área de distribución de lá especie en esta población. Para ello, sobre la base topográfica 1:10.000, se delimitó un área de $9 \mathrm{Km}^{2}$ (900 ha), sobre la que se superpuso una malla de cuadrículas de $100 \mathrm{~m}$ de lado (un total de 900 cuadrículas) y se evaluó la presencia o ausencia de la especie y la cobertura de suelo que presentaba.

\subsubsection{Densidad de individuos en las distintas coberturas del suelo}

En cada cuadrícula de $100 \times 100 \mathrm{~m}$ con presencia de la especie se realizaron 20 transectos de $160 \mathrm{~m}$ de longitud y $10 \mathrm{~m}$ de ancho, que representan un total de 16 cuadriculas de $10 \times 10 \mathrm{~m}$ (1.6 ha), de forma que pudieran detectarse las discontinuidades o manchas ocupadas por individuos de $V$. lucentina. En cada cuadrícula se contó el número de individuos de la especie y se clasificaron por clases de altura. La Clase 0 corresponde a las plántulas $(0.5-1 \mathrm{~cm})$, la Clase 1 a los individuos con alturas entre 5 y $10 \mathrm{~cm}$, la Clase 2 entre 10 y $20 \mathrm{~cm}$, y la Clase 3 , entre 20 y $30 \mathrm{~cm}$.

Otras variables que se tuvieron en cuenta fueron la pendiente de la parcela $(\%)$, el recubrimiento total de vegetación (\%), la altitud (m) y la cobertura de suelo, agrupada en los siguientes tipos: a) ladera (principalmente afloramientos rocosos), b) camino (márgenes o cunetas), c) repoblación de $P$. halepensis, d) cultivo abandonado en bancales, y e) espartal (comunidad dominada por la especie Stipa tenacissima).

Para comprobar qué factores podían afectar de forma significativa al número de individuos de $V$. lucentina por unidad de superficie, se compararon las distintas densidades obtenidas mediante el análisis múltiple de la varianza. La variable dependiente utilizada ha sido el número de individuos en cada ambiente y las covariables, la pendiente y la altitud de las parcelas. El test a posteriori utilizado fue el de Bonferroni, para distintos tamaños de muestra, con el que se estableció cuales eran las variables que mostraban diferencias significativas (Sokal y Rohlf, 1995). El análisis se realizó considerando el total de individuos y las distintas clases de tamaño.

\subsubsection{Análisis de la distribución de individuos en la población del Bec de L'Aguila}

Utilizando las densidades obtenidas en las cuadrículas, se analizó la distribución de individuos en el área donde está presente la especie y con el fin de definir áreas 
de mayor y menor densidad. La cartografía de las densidades de individuos totales y para cada una de las clases de tamaños se ha realizado calculando las isolíneas de densidad con el método de interpolación lineal kriging, mediante autocovarianzas aplicadas a la red de parcelas muestreadas (Rossi et al. 1992).

\section{4. 3. Relación entre los patrones de distribución y las clases de tamaño}

La relación entre las parcelas con mayor densidad de individuos y la distribución de individuos en clases de tamaño, se realizó utilizando el coeficiente de variación y el índice de uniformidad Pielou (E), (Magurran, 1989):

\section{Donde:}

$\mathrm{H}^{\prime}=$ índice de diversidad (Shannon-Weaver) de clases de tamaño

$\mathrm{H}_{\max }=$ indice de diversidad máximo

$S=$ número total de clases de tamaño $=4$

ni $=$ número de individuos en la clase de tamaño i

$\mathrm{N}=$ número de individuos en todas las clases de tamaño

Las correlaciones entre el número total de individuos, número de clases de tamaño ocupados, la variabilidad y la uniformidad de abundancia total de individuos en cada clase se realizó utilizando una matriz de correlación mediante el test de rangos de Spearman. Este análisis se efectuó en las dos escalas corológicas, utilizando los resultados de las tres poblaciones (macro) y evaluando solo la población del Bec de l'Aguila (meso).

\section{Resultados}

\subsection{Distribución espacial regional: Macro-escala}

La superficie ocupada por las tres poblaciones es inferior a $12 \mathrm{~km}^{2}$. El área de distribución potencial de Vella lucentina en la población de Bec de l'Aguila es de $9 \mathrm{~km}^{2}$, en la Sierra de los Tajos de $1 \mathrm{~km}^{2}$ y en La Alcoraya, de $2 \mathrm{~km}^{2}$ (Figura 4). Este primer análisis descriptivo parece indicar una disgregación entre poblaciones. La distancia que separa a la población de Bec de l'Aguila y la sierra de los Tajos es de $6 \mathrm{~km}$; entre la Sierra de los Tajos y La Alcoraya hay $10 \mathrm{~km}$; y entre La Alcoraya y Bec de l'Aguila, $15 \mathrm{~km}$. Entre dichas poblaciones no existe continuidad en la distribución de las plantas. 


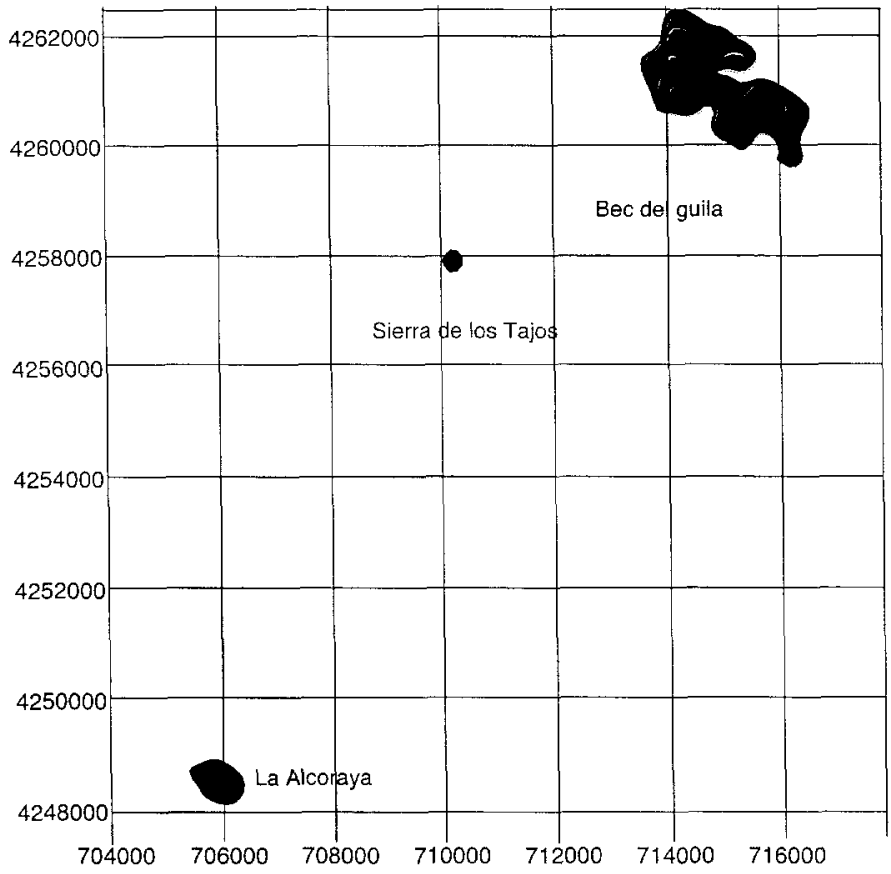

Figura 4. Área de distribución potencial de las tres poblaciones de Vella lucentina identificadas. Análisis a macro-escala obtenido mediante interpolación lineal de los puntos de muestreo (coordenadas UTM).

\subsection{Distribución espacial local: Meso-escala}

La discontinuidad de $V$. lucentina en la población de Bec de l'Aguila se hace patente al observar que está presente tan sólo en un 28\% del total de las cuadrículas. Esto permite reducir el área de distribución de la especie en esta población a $2.56 \mathrm{~km}^{2}(256 \mathrm{ha})$. De las categorías de coberturas de suelo presentes en el área de distribución el ambiente que ocupa un área mayor es la ladera, con 168 ha, mientras que las repoblaciones ocupan 25 ha. El ambiente afectado por los márgenes de camino ha sido estimado en 23 ha, mientras que los espartales ocupan 11 ha y los bancales con cultivos abandonados se restringen a 7 ha. El suelo urbanizado es la categoría con menor superficie, con tan sólo $26.3 \cdot 10^{-6}$ ha.

\subsubsection{Densidad de individuos}

Se contabilizan un total de 3874 individuos, de los que un $63 \%$ se encuentran en la ladera. El 19\% de los individuos viven sobre bancales abandonados, mientras que 
en el borde del camino se encuentran un 10\%, y un $8 \%$ en la repoblación. En el espartal, la presencia de individuos es prácticamente nula (Tabla 1).

La densidad de individuos varía significativamente entre las coberturas de suelo. Los ambientes analizados presentan diferencias significativas en cuanto a la densidad de individuos de esta especie $(\mathrm{p}<0.01)$. Por el contrario, la altitud y la pendiente no condicionan la presencia de los individuos de V. lucentina (Tabla 2).

Si se tiene en cuenta la densidad de individuos entre los diferentes tipos de cobertura del suelo se observa que, a pesar de existir diferencias significativas, los ambientes ladera y borde de camino tienen una densidad de magnitud similar, entre 53 y 47 ind $0.01 \mathrm{ha}^{-1}$ respectivamente. La repoblación, los bancales y las zonas de espartal tienen densidades menores. En la repoblación la densidad es de 5 ind $\cdot 0.01 \mathrm{ha}^{-1}$, en los bancales 14 ind $\cdot 0.01 \mathrm{ha}^{-1}$, mientras que en el espartal no se observó ningún individuo de la especie (Tabla 1).

Tabla 1. Número de individuos totales y valores medios de Vella lucentina en parcelas de $10 \times 10$ $\mathrm{m}$ sobre las diferentes coberturas de suelo. Cobertura de la vegetación y altitud y pendiente media de la parcela.

\begin{tabular}{|lcccccc|}
\hline $\begin{array}{c}\text { Coberturas } \\
\text { de stuelo }\end{array}$ & $\begin{array}{c}N \\
\text { Parcelas }\end{array}$ & $\begin{array}{c}\text { Pendiente } \\
\text { media }(\%)\end{array}$ & $\begin{array}{c}\text { Altitud } \\
\text { media }(m)\end{array}$ & $\begin{array}{c}\text { Cobertura } \\
\text { media (\%) }\end{array}$ & $\begin{array}{c}N^{\circ} \text { total } \\
\text { ind }\end{array}$ & $\begin{array}{c}N^{\circ} \text { medio } \\
\text { ind }\end{array}$ \\
\hline Bancal & 38 & 0.3 & 332 & 46.5 & 589 & 14.09 \\
Camino & 12 & 23.7 & 325 & 36.3 & 551 & 47.83 \\
Espartal & 10 & 62.0 & 402 & 68.5 & 0 & 0.00 \\
Ladera & 50 & 33.1 & 365 & 31.1 & 2647 & 53.14 \\
Repobl. & 10 & 5.0 & 220 & 25.0 & 87 & 10.06 \\
\hline
\end{tabular}

Tabla 2. Comparación del número de individuos en las distintas coberturas de suelo. Análisis múltiple de la varianza, intervalo de confianza $95 \%$ ( $n=120$ parcelas).

\begin{tabular}{|ccccc|}
\hline & Suma cuadrados & $g . l$ & $F$ & Signific. \\
\hline Factor & & & & \\
$\quad \begin{array}{c}\text { Coberturas } \\
\text { Covariables }\end{array}$ & 37885.2 & 4 & 5.65 & 0.00 \\
$\quad \begin{array}{l}\text { Pendiente } \\
\text { Altitud }\end{array}$ & 470.5 & 1 & 0.35 & 0.55 \\
& 1671.2 & 1 & 1.25 & 0.26 \\
\hline
\end{tabular}

\subsubsection{Análisis de la distribución de individuos en la población del Bec de L'Aguila}

Aproximadamente un $80 \%$ de los individuos analizados tiene tamaños entre 10 y $20 \mathrm{~cm}$. Solo un $2 \%$ de los individuos eran plántulas y un $9 \%$ correspondería a individuos con un tamaño mayor de $30 \mathrm{~cm}$ altura. La densidad de individuos adultos, con 
alturas entre $5-30 \mathrm{~cm}$, es de 2 ind $\cdot \mathrm{m}^{-2}$, en las plántulas es mucho menor $\left(0.1 \mathrm{ind} \cdot \mathrm{m}^{-2}\right)$, y la densidad de los individuos de mas de $30 \mathrm{~cm}$ de altura es de 0.47 ind $\cdot \mathrm{m}^{-2}$.

La densidad de individuos de las distintas clases de tamaño es diferente en cada una de las coberturas de suelo analizadas ( $\mathrm{p}<0.05$, gl=3, Tabla 3), con excepción de las plántulas. El número de plántulas es semejante en todas las categorías de cobertura del suelo 1 ( $P>0.05$, Tabla 3).

Tabla 3. Comparación entre el número de individuos de las distintas clases de tamaño en las categorias de cobertura de suelo más representativas. Valores medios y error estándar. La letra sobrescrita indica las diferencias significativas entre grupos $(p<0.05)$.

\begin{tabular}{|c|c|c|c|c|c|}
\hline $\begin{array}{c}N^{o} \text { individuos } \\
\text { Clase de altura }(\mathrm{cm})\end{array}$ & $\begin{array}{l}\text { Coberturas } \\
\text { de suelo }\end{array}$ & $\begin{array}{l}\text { Valor } \\
\text { medio }\end{array}$ & $\begin{array}{l}\text { Error } \\
\text { eständar }\end{array}$ & $F$ & Significacion \\
\hline \multirow{5}{*}{ Plántulas } & Bancal & 0 & 0.00 & \multirow[t]{5}{*}{1.68} & \multirow[t]{5}{*}{0.16} \\
\hline & Camino & 1.83 & 1.03 & & \\
\hline & Espartal & 0 & 0.00 & & \\
\hline & Ladera & 2.31 & 0.98 & & \\
\hline & Repoblación & 0.30 & 0.30 & & \\
\hline \multirow{5}{*}{$10-15$} & Bancal & $8.68 \mathrm{~b}$ & 2.95 & \multirow[t]{5}{*}{6.22} & \multirow[t]{5}{*}{0.0001} \\
\hline & Camino & $22.50 \mathrm{a}$ & 6.37 & & \\
\hline & Espartal & 0 & 0.00 & & \\
\hline & Ladera & $29.68 \mathrm{a}$ & 4.88 & & \\
\hline & Repoblación & 0.9 & 0.60 & & \\
\hline \multirow{5}{*}{$15-20$} & Bancal & $5.34 \mathrm{~b}$ & 1.5 & \multirow[t]{5}{*}{12.37} & \multirow[t]{5}{*}{0.0001} \\
\hline & Camino & $17.08 \mathrm{a}$ & 3.30 & & \\
\hline & Espartal & 0 & 0.00 & & \\
\hline & Ladera & $18.91 a$ & 1.91 & & \\
\hline & Repoblación & $3.90 \mathrm{~b}$ & 2.96 & & \\
\hline \multirow{5}{*}{$>20$} & Bancal & $1.47 \mathrm{~b}$ & 0.58 & \multirow[t]{5}{*}{3.07} & \multirow[t]{5}{*}{0.019} \\
\hline & Camino & $4.50 \mathrm{a}$ & 1.01 & & \\
\hline & Espartal & 0 & 0.00 & & \\
\hline & Ladera & $4.22 \mathrm{a}$ & 0.63 & & \\
\hline & Repoblación & $3.60 \mathrm{~b}$ & 3.28 & & \\
\hline
\end{tabular}

El porcentaje de individuos de cada clase de tamaño respecto al total de individuos muestreados, presenta el mismo patrón en las distintas categorías. Por tanto, las clases que se encuentran menos representadas son las plántulas y los individuos adultos, mientras que las clases con más individuos son las de tamaños intermedios (Figura 3).

La densidad obtenida en cada una de las parcelas de $100 \mathrm{~m}^{2}(0.01 \mathrm{ha})$ se ha utilizado para establecer las isolineas de densidad en el área de distribución de la especie (Figura 5). La especie Vella lucentina es abundante localmente, y se pueden observar ciertos patrones en la distribución del número de individuos. Aparecen zonas que podrían considerarse núcleos, ya que presentan el mayor número de indi- 
viduos, y conforme nos alejamos de estas disminuye la densidad (Figura 5). Los núcleos de mayor densidad se localizan en las coordenadas UTM, 1( $\mathrm{X}_{\text {min }} 714700, \mathrm{Y}_{\mathrm{w}, \mathrm{n}}$ 4260500; $\left.X_{\max } 715200, Y_{\max } 4260500\right), 2 X_{\min } 715200, Y_{\min } 4260900 ; X_{\max } 716000, Y_{\max }$ 4260500) y $3\left(X_{\min } 713500, Y_{\min } 4261500 ; X_{\min } 713800, Y_{\min }\right.$ 4261700). Se puede observar que en los años 1999 y 2000 , cuando se realizó el muestreo de individuos, no se contabilizó prácticamente ninguna germinación en toda el área de muestreo, excepto en la zona de coordenadas 30SYH5360. Los individuos con alturas entre 5 y $20 \mathrm{~cm}$ son los más abundantes y los que se encuentran más distribuidos por el territorio (Figuras 6.b y 6.c). Los individuos pertenecientes a las clases de tamaño mayores, son abundantes y se encuentran restringidos en un área determinada por las coordenadas UTM 07248004260800 .

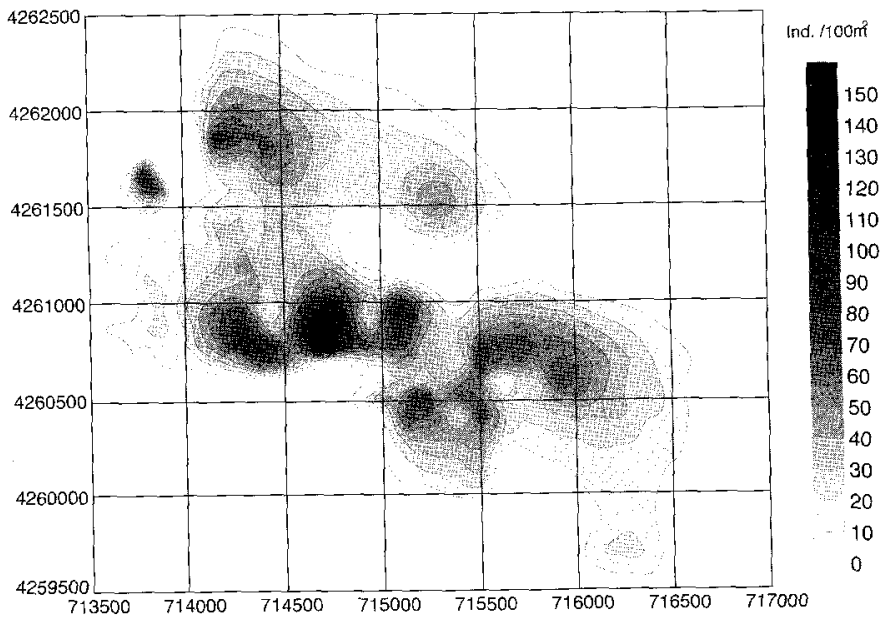

Figura 5. Densidad de individuos $\left(n^{\circ}\right.$ ind $\left./ 100 \mathrm{~m}^{2}\right)$ de Vella lucentina. Se presenta la distribución del total de individuos incluyendo todas las clases de tamaño analizadas. Resultado del método de interpolación lineal kriging (coordenadas UTM).

\subsubsection{Relación entre los patrones de distribución y lảs clases de tamaño}

El análisis de variación de individuos en cada clase de tamaño entre las tres poblaciones (macro-escala), sugiere que en las parcelas que poseen un mayor número de clases de tamaños se muestran menos variables en el número de individuos en cada clase $(r$ Spearman $=-0.67, p<0.001)$ y por tanto mas equilibradas, evidenciado una mayor uniformidad en la proporción de los individuos de cada clase con respecto a la abundancia total ( $\mathrm{r}$ Spearman $=0.79, \mathrm{p}<0.001$ ). A su vez, se observa una leve tendencia a que parcelas con un mayor número de individuos posean un mayor número 
de clases $(r$ Spearman $=0.48, p<0.001$ ). Si bien la relación entre el número total de individuos con el coeficiente de variabilidad y la equidad es negativa y positiva respectivamente, parece no existir una correlación discernible pues el valor del índice de Spearman en menor a 0.3 (Tabla 4).

El análisis de variación de individuos en cada clase en la población del Bec de l'Aguila (mesoescala), muestra la misma tendencia que el análisis anterior (macroescala), con la única diferencia que la intensidad de las correlaciones con el número de clases es algo mas baja, principalmente cuando se relaciona esta última con el número de individuos totales (Tabla 5).

Tabla 4. Análisis de variación de individuos en las tres poblaciones (macro-escala). Matriz de Correlación de Spearman entre: número de clases ( $n^{\circ}$ clases), uniformidad (índice de Pielou), número total de individuos (Total) y coeficiente de variación (C.V.), para un total de 88 parcelas.

\begin{tabular}{|l|c|c|}
\cline { 2 - 3 } \multicolumn{1}{c|}{} & r Spearman & Significación \\
\hline$N^{\circ}$ Clases vs Total & 0.478 & 0.0000 \\
$N^{\circ}$ Clases vs C.V. & -0.668 & 0.0000 \\
$N^{\circ}$ Clases vs Uniformidad & 0.794 & 0.0000 \\
Total vs C.V. & -0.219 & 0.0408 \\
Total vs Uniformidad & 0.294 & 0.0055 \\
Uniformidad vs C.V. & -0.969 & 0.0000 \\
\hline
\end{tabular}

Tabla 5. Análisis de la variación de individuos en la población del Bec de l'Aguila (meso-escala). Matriz de Correlación de Spearman entre: número de clases ( $n^{\circ}$ clases), uniformidad (índice de Pielou), número total de individuos (Total) y coeficiente de variación (C.V.), para un total de 70 parcelas.

\begin{tabular}{|l|c|c|}
\cline { 2 - 3 } \multicolumn{1}{c|}{} & r Spearman & Significación \\
\hline$N^{\circ}$ Clases vs Total & 0.291 & 0.0146 \\
$N^{\circ}$ Clases vs C.V. & -0.537 & 0.0000 \\
$N^{\circ}$ Clases vs Uniformidad & 0.709 & 0.0000 \\
Total vs C.V. & -0.268 & 0.0247 \\
Total vs Uniformidad & 0.316 & 0.0077 \\
Uniformidad vs C.V. & -0.957 & 0.0000 \\
\hline
\end{tabular}

\section{Discusión y conclusiones}

El estudio de los patrones espaciales en las comunidades de plantas, permite comprender determinados procesos de la dinámica de sus poblaciones, como la dispersión y las interacciones intra o interespecíficas, e incluso puede determinar la estructura genética y evolución de las poblaciones (Hanski, 2000). A través de la estructura espacial se pueden observar asociaciones históricas entre poblaciones, así como los 
sucesos de aislamiento que han dado lugar a distintas formas de distribución de especies vegetales (Thompson, 1999). En la especie Vella lucentina, la estructura espacial es importante, en primer lugar, por tratarse de una especie endémica y en segundo lugar, por las restricciones que impone el hábitat semiárido donde se encuentra. Por lo tanto el estudio de los patrones de distribución puede resultar clave para determinar procesos de colonización y extinción o incluso la especificidad por determinados usos de suelo.
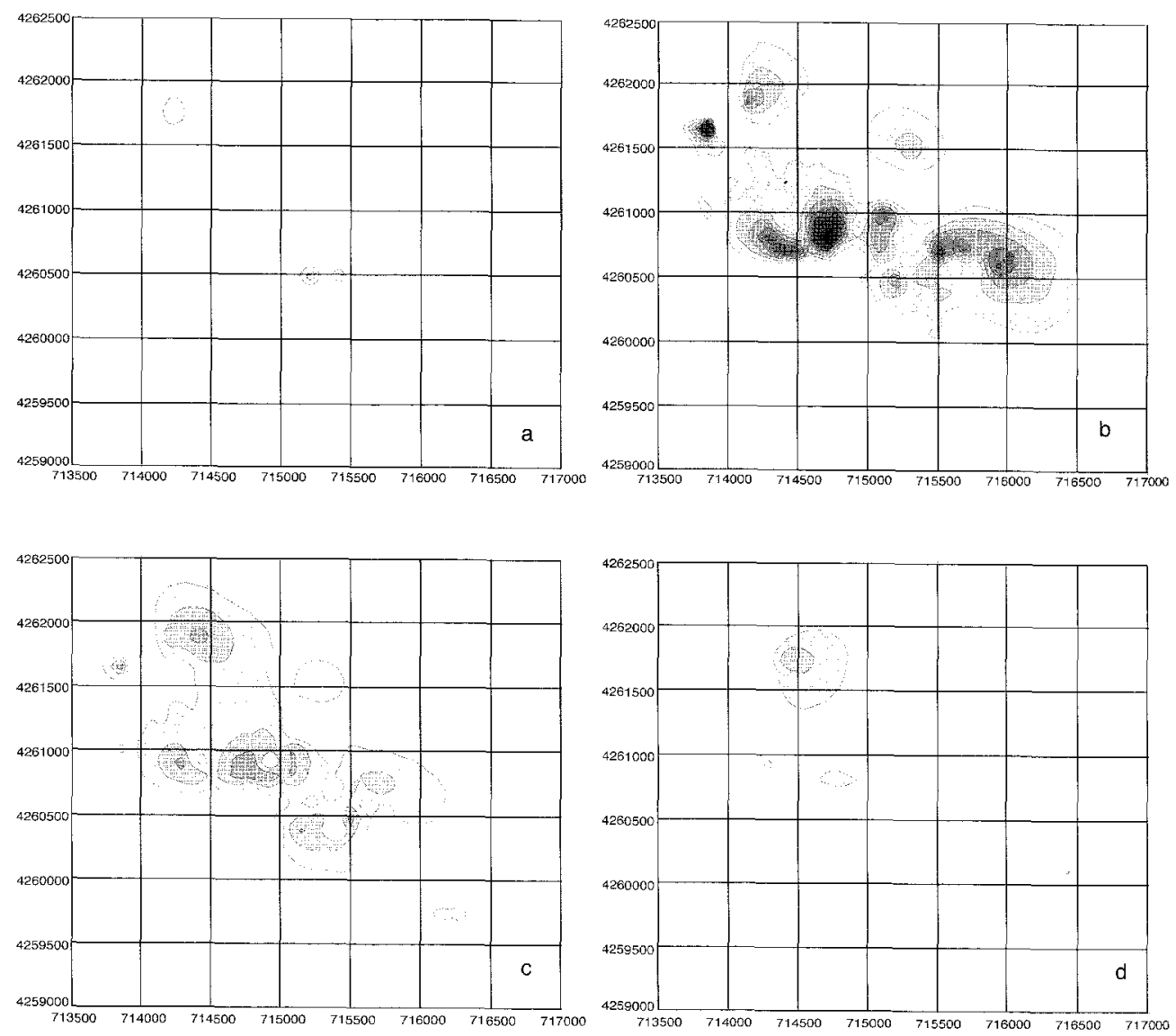

Figura 6. Distribución de la densidad de individuos (ind. $100 \mathrm{~m}$-2) para las distintas clases de tamaño identificadas: a) plántulas (Clase 0), b) individuos de 2-10 cm altura (Clase 1), c) individuos de $10-20 \mathrm{~cm}$ altura (Clase 2), d) individuos de $20-30 \mathrm{~cm}$ altura (Clase 3 ). El sistema de referencia esta basado en las coordenadas UTM. 
En los últimos 10 años se ha afianzado la idea de que las comunidades de plantas en clima semiárido a menudo muestran una dinámica compleja (Westoby et al., 1989; Fiedler y Jain, 1992; Walker, 1993; Milton, 1994) que envuelve procesos no lineares dominados por eventos estocásticos o azarosos (Westoby et al., 1989). De esta forma la estructura de la vegetación generalmente cambia en función de eventos episódicos e impredecibles (Milton, 1995). Wiegand y Milton (1996) encontraron que la secuencia de eventos de precipitación, estocásticos y poco predecibles, influenciaba la comunidad de plantas en una escala de tiempo corto. En la zona de estudio, y en el ámbito de la Comunidad Valenciana también se dan estos eventos de precipitación, que se definen como de carácter extremo e impredecible (De Luís, 2000). Nuestros estudios han corroborado la idea de en estas zonas semiáridas, en las que las especies vegetales dependen en gran medida de los factores abióticos, un único evento de germinación y establecimiento de plántulas puede determinar la distribución y dinámica de la especie por muchos años. Así durante el periodo de estudio 1997-99, en la población del Bec de L'Aguila, se detectaron nuevos reclutamientos para la población de Vella lucentina en Octubre de 1997 y Enero de 1998, justo a continuación de los meses más lluviosos (Figura 1) (Gras, 2001).

Vella lucentina se puede considerar una especie localmente abundante, de ámbito geográfico restringido y con una especialización de hábitat en las coberturas de suelo denominadas ladera, aunque con preferencias locales hacia el margen de camino que definen ciertamente su rareza. Gaston (1994) y Kunin (1997) sugieren tres mecanismos principales causantes de la rareza: la especialización ecológica, la falta de dispersión y las contingencias históricas. En el caso de la especie analizada, y respecto al primer mecanismo, podemos calificar la rareza de $V$. Lucentina de elevada, pues tan sólo se desarrolla en ambientes semiáridos con baja competencia. Por otra parte, la especie, que presenta poca capacidad de dispersión en el espacio, colonizaría primordialmente lugares disponibles cercanos a las plantas adultas. En Vella lucentina las distancias máximas de dispersión han sido de $80 \mathrm{~cm}$ pero la mayor densidad de plántulas se ha encontrado a distancias entre 5 y $20 \mathrm{~cm}$ de la planta adulta (Gras, 2001). Por tanto, la falta de eventos de precipitación que hayan hecho posible la dispersión a distancias largas y la eliminación de la especie en determinadas zonas puede haber condicionado su patrón espacial y la distribución en el futuro (Gras, 2001).

A escala regional, las poblaciones de $V$. lucentina aparecen fragmentadas. El conocimiento actual de esta especie sólo nos permite barajar ciertas hipótesis sobre las causas de la fragmentación de las áreas ocupadas. Cabe señalar que el hecho de que actualmente presente un área de distribución restringida puede ser debido a eventos naturales históricos, como ha sucedido con otras especies paleoendémicas (Gómez-Campo, 1999), donde la estructura espacial es el resultado de determinados eventos de aislamiento geográfico durante el Mioceno y Plioceno (Padilla y Ramón, 1997). Por otra parte, no se puede descartar un origen natural de la estructura fragmentada de esta especie. 
El área de distribución de la especie se encuentra expuesto a la influencia humana y al desarrollo de actividades que han limitado la expansión de la especie, como ha ocurrido en otras especies raras (Pavlovic y Bowles, 1996). En este caso, la fragmentación podría haber sido causada por la existencia de núcleos de población, por la creación de redes viarias y también debido al cultivo extensivo de las zonas que rodean a las tres poblaciones (Santa Creu, 2000).

En la población analizada, la especie es abundante localmente, 31.49 ind $0.01 \mathrm{ha}^{-1}$, aunque la densidad de individuos varia en las distintas coberturas de suelo. Dentro de la zona que potencialmente podría constituir su hábitat, es más abundante en tipos de cobertura del suelo poco alteradas como las de ladera ( 53.14 ind $0.01 \mathrm{ha}^{-1}$ ), si bien cabe destacar la presencia de la especie en los márgenes de caminos ( 47.8 ind $0.01 \mathrm{ha}^{-1}$ ). En general se observa una tendencia de la especie a ocupar zonas en las que la vegetación es escasa y se encuentra dispersa. Así, ambientes con porcentajes de cobertura de otras especies de un $60 \%$ o un $68 \%$, como es en el que se desarrolla Stipa tenacissima (espartal) y en las repoblaciones (Pinus halepensis), no presentan ningún individuo de $V$. lucentina o el número de individuos es muy bajo. Además cabe señalar que el método de preparación del terreno utilizado en las repoblaciones es muy agresivo (subsolado lineal), lo cual podría influir sobre la escasa presencia de individuos de la especie, ya que podrían haber sido eliminados parcialmente con el laboreo. En el borde de camino el porcentaje de cobertura es de un 41\%, semejante al de los bancales donde es de un $45 \%$, sin embargo ambos presentan diferencias en cuanto al número de individuos de $V$. lucentina.

En zonas levemente perturbadas como son los márgenes de camino, el número de individuos es mayor que en las zonas que habían sido cultivadas. Podemos aventurar dos posibles hipótesis al respecto: a) el muro de sillería de los bancales presenta una barrera para la dispersión postdiseminativa de la especie, que utiliza la arroyada como vector de transporte, y b) gran parte de la cobertura total en los bancales está generada por la especie Brachypodium retusum, una graminea con gran capacidad de extensión lateral y colonización, ausente en los márgenes de camino, que podría, eventualmente, desplazar a $V$. lucentina por competencia. La mayor densidad en las cunetas permite sugerir que dicho hábitat es apropiado para la especie, ya que parece ser que las pequeñas perturbaciones que implican una ligera remoción del suelo no afectan a la supervivencia de la especie (Gras, 2001), mientras que pueden eliminar posibles especies competidoras. Por otra parte, estos ambientes reciben una mayor disponibilidad de recursos hídricos, especialmente en aquellos caminos que cortan laderas donde existen taludes, aumentando la superficie de la microcuenca de captación de agua de precipitación (De Simón, 1990), favoreciendo así el desarrollo y crecimiento de estas plantas.

Al analizar toda el área de distribución, se observa que la densidad los individuos varía con el tamaño de éstos, ya que el área de distribución de plántulas y adultos es 
muy restringida. La clase de altura más abundante es la intermedia, entre 10 y $20 \mathrm{~cm}$. En 1999, la densidad de plántulas fue escasa y su distribución confinada a una única zona debido a la combinación de factores como la falta de individuos reproductivos o semillas, o de agentes que dispersen las semillas (Gras, 2001). Otra limitación puede ser, el hecho de que no existan lugares adecuados para que germinen las semillas (Eriksson y Ehrlén, 1992; Tilman, 1997), al igual que se ha descrito en especies de árboles la tendencia de las semillas pequeñas a establecerse en lugares específicos, micrositios (microsites), constituidos por afloramientos rocosos y con poco suelo disponible (Prentice y Wegner, 1985; Nakashizuka, 1989; Grubb, 1985). La heterogeneidad observada en la estructura de tamaños de las subpoblaciones podría entonces asociarse a la distribución irregular en la disponibilidad de dichos micrositios.

En la población de Bec de l'Aguila, existen zonas con distinta densidad de individuos. En general, la estructura espacial de los individuos se encuentra formando núcleos de mayor densidad (Figura 5) situados en las coordenadas UTM 30SYH4760, 30 SYH5260 y 30 SYH3761. Este patrón es debido a distintos factores, y a la combinación de los aspectos morfológicos (crecimiento y tamaño de la especie), ambientales y fitosociológicos (Little y Dale, 1999). El resultado de ello es un conjunto metapoblacional (observable en la Figura 4 y siguientes). Las subpoblaciones con una elevada densidad de plantas tienden a presentar un mayor número de clases de tamaños, las mismas que presentan estructuras demográficas más equilibradas y actúan como fuentes de diásporas (semillas), mientras que aquellas con menores densidades actuarían como sumideros (Hanski y Gilpin, 1991). Para favorecer la conservación de las pocas poblaciones de la especie $V$. lucentina cabría por tanto, optimizar los esfuerzos hacia la preservación de las subpoblaciones fuente, localizadas en la cartografía, pues permitirian mantener una producción de semillas que facilitaría el mantenimiento de la viabilidad de la población, con el posterior reclutamiento de individuos juveniles, si las condiciones ambientales (principalmente las precipitaciones primaverales) permitieran su germinación y crecimiento.

\section{Bibliografía}

Brown, J. H., Mehlman, D. W. y Stevens, G.C. (1995): Spatial variation in abundance. Ecology, 76, p. 2028-2043.

Caswell, H. y Cohen, J.E. (1991): Communities in patchy environments: a model of disturbance, competition, and heterogeneity. En Kolasa, J. y Pickett, S.T.A. (eds)
Ecological beterogeneity. Nueva York, Springer-Verlag.

Crespo, M.B. (1992): A new species of vella L. Brassicaceae from the south-eastern part of Iberian Peninsula. Biological Journal of the Linnean Society, 109, p. 369-376. 
Crespo, M.B., Lledó, M.D., Fay, M.F., y Chase, M.W. (2000): Subtribe Vellinae Brassiceae, Brassicaceae: a combined analysis of ITS nrDNA sequences and morphological data. Annals of Botany, 86, p. 53-62.

Dale, M.R.T.(1999): Spatial pattern analysis in plant ecology. En Birks, H.J. B. y Wiens, J.A.(eds). Cambridge University Press.

De Luis, M. (2000): Estudio Espacial y Temporal de las Tendencias de Lluvia en la Comunidad Valenciana 1961-1990. Logroño, Geoforma.

De Simón, E. (1990): Restauración de la vegetación en cuencas mediterráneas: repoblaciones en zonas áridas. Ecologia, p. 401-427.

Dramstad, W.E., Ison, J.D. y Forman, R.T.T. (1996): Landscape ecology principles in ladscape arcbitecture and land-use planning. Harvard University, Graduate school of design.

Eriksson, O. (2000): Seed dispersal and colonization ability of plants- Assessment and implications for conservation. Folia Geobotanica, 35, p. 115-123.

Eriksson, O. y Ehrlén, J. (1992): Seed and microsite limitation of recruitment in plant populations. Oecologia Berlin, 91, p. 360-364.

Fahrig, L. y Merriam, G. (1994): Conservation of fragmented population. Conservation biology, 8, p. 50-59.

Fiedler, P.I., Jain, S.K.(1992): Conservation biology: The theory and practice of Nature Conservation and Managament. Nueva York/Londres, Chapmant \& Hall.

Gaston, K. J. (1994): Rarity. Londres, Chapman \& Hall.

Gilpin, M. E. (1987): Spatial structure and population viability. En Soulé, M.E. (ed).
Viable populations for conservation. Massachussets, USA. Sinauer, Sunderland

Gómez-Campo, C. (1999): Seedless and seeded beak in the tribe. Brassiceac Eucarpia Cruciferae Newsl, 21, p. 11-12.

Gras, M.J. (2001): Ecología de la conservacion de un endemismo exclusivo de la provincia de Alicante: Vella lucentina M.B. Crespo. Estudio en ambientes contrastados. Universidad de Alicante. Tesis de licenciatura (inédito).

Greuter, W., Burdet, H.M., y Long, G. (1986): Conservatoire et jarden botaniques. Ginebra.

Grubb, PJ. (1985): Plant population and vegetation in relation to habitat, disturbance and competition: problems of generalization. En White, J. y Junk, W. (eds) Handbook of vegetation science 3. The population structure of vegetation. Ia Haya (Paîses Bajos), p. 595-622.

Hanski, I. (1994): Spatial Scale, Patchiness and Population Dynamics on Land. Pbilos. Trans. R. Soc Lond, 343, p. 19-25.

Hanski, I. (1998): Metapopulation dynamics. Nature London, 396, p. 49.

Hanski, I. y Gilpin, M. (1991): Metapopulation dynamics: a brief history and conceptual domain. Biological Journal of the Linnean Society, 42, p. 3-16.

Hanski, I., Alho, J., y Moilanen, A. (2000): Estimating the parameters of survival and migration of individuals in metapopulations. Ecology, 81, p. 239-251.

IGME (Instituto geológico minero de España) (1978): (871). E. 1: 50000 . Madrid, Servicio de publicaciones del ministerio de industria.

Kunin, W. E. (1997): Introduction: on the causes and consequences of rare-common differences. En Kunin, W. E. y Gaston, K. 
J. (eds) The biology of rarity. Causes and consequences of rare-common differences. Londres. Chapman y Hall, p. 3-11.

Laguna, E. (1994): Introducción. En: Aguilella, A., (eds) Flora vascular rara, endémica o amenazada de la Comunidad Valenciana. Valencia, Generalitat Valenciana. Consellería de Medio Ambiente,

Laguna, E. (1995): Microrreservas de flora: un nuevo modelo de conservación en la Comunidad Valenciana. Quercus, p. 22-26.

Laguna, E. y Crespo, M.B. (1996): Asignación de las nuevas categorías UICN a la flora endémica de la Comunidad Valenciana. En VV.AA, Tomo extraordinario. 125 Aniversario de la RSEHN, p. 385-387.

Laguna, E., Crespo, M.B., Mateo-Sanz, G., Lopez, S., Fabregat, C., Herrero-Borgonon, J.J., Carretero, J.L., Aguileila, A., y Figuerola, R. (1998): Flora endémica rara o amenazada de la Comunidad Valenciana. Valencia. Generalitat Valenciana, Consellería de Medio Ambiente.

Lande, R. 1988. Genetics and demography in biological conservation. Science, 241, p. $145-146$.

Little, L.R. y Dale, M.R.T. (1999): A method for analysing spatio-temporal pattern in plant establishment, tested on a Populus balsamifera clone. Journal of Ecology, 87, p. 620-627.

Lledó, M.D., Crespo, M.B., del Amo, J.B. (1995): In vitro multiplication of Vella lucentina M.B. Crespo (Brassicaceae), a Spanish threatened endemic. In Vitro Cell.Develop.Biol.Pla, 31, p. 199-201.

Magurran, A. (1989): Diversidad ecológica y su medición. Barcelona, Vedrá.

Menaut, J.C., Gignoux, J., Prado, C., Clobert, J. (1990): Tree communityr dynamics in a humid savanna of Cote d'Ivore: modelling the effects of fire an compettition with grass and neighbors. Joumal of biogeography, 17, p. 471-481.

Menges, E. S. (1990): The application of minimum viable population theory to plants. Genetics and Conservation of Rare Plants, p. $46-61$.

Milton, S.J. (1994): Growth, florwering and recruiment of shrubs in grazed and in protected rangeland in the arid Karoo, South Africa. Vegetatio, 111, p. 17-27

Milton, S.J. (1995): Spatial and temporal patterns in the emergence and survival of seedlings in arid Karoo shrubland. Journal of Applied Ecology, 32, p. $145-156$.

Montaña, C. (1992): The colonization of bare areas in two phase mosaics of an arid ecosystem. Journal of Ecology, 80, p. 315-327.

Naito, K. y Nakagoshi, N. (1995): The conservation ecology of Iris rossii Baker Iridaceae, a threatened plant in rural Japan. J. Plant Res, 108, p. 477-482.

Nakashizuka, T. (1989): Role of uprooting in composition and dynamics of an oldgrowth forest in Japan. Ecology, 70, p. $1273-1278$.

Nakashizuka, T. y Numata, M. (1982): Regeneration process of climax beech forests I. Structure of a beech forest with the undergrowth of Sasa. Jpn.J. Ecol., 32, p. 57-67.

Padilla, A. y Ramón, A. (1997): Planeamiento ambiental a escala de detalle: microrreservas de flora en la Comunidad Valenciana, Imestigaciones Geográficas, 14, p. 117-128.

Pavlovic, N.B. y Bowles, M.L. (1996): Rare plant monitoring at Indiana Dunes National Lakeshore. En: Halvorson, W.L. y Davis, G. E. (eds). Science and Ecosystents Management in the National Purks. Tucson, The University of Arizona Press, p. 253-280. 
Pérez-Cueva, A. (1994). Atlas climàtic de la Comunitat Valenciana Conselleria d'Obres Públiques, Urbanisme i Transports. Valencia, Generalitat Valenciana, Collecció Territori, $\mathrm{n}^{\circ} 4$.

Prentice, I.C. y Wegner, M.J.A. (1985): Clump spacing in a desert dwarf shurb community. Vegetatio, 63, p. 133-139.

Ravinowitz, D. (1981): Seven forms of rarity. En Synge, $H$. (ed) The biological aspects of rare plant consenation. Chischester (RU), Wiley, J.

Ravinowitz, D. (1986): Seven forms of rarity and their frecuency in the flora of the British Isles. En Soule, M. (ed). Conservation biology: the scarcity and diversity. Massachusetts (USA), Sinauer, Sunderland.

Rey Benayas, J. M., Scheiner,S.M., García Sânchez-Colomer, M. y Levassor, C. (1999): Commonness and rarity: theory and application of a new model to Mediterranean montane grasslands. Conservation Ecology, 3 (1), p. 5.

Rivas-Martínez, S. (1983): Pisos bioclimáticos de España. Lazaroa, $n^{\circ} 5$.

Rossi, R., Mulla, D., Journel, A., y Franz, E. (1992): Geostatistical tools for modeling and interpreting ecological spatial dependence. Ecological Monographs, 62, p. 277-314.

Sainz, H., Franco, F. y Arias, J. (1996): Vella pseudocytisus subsp. Paut. Estrategias para la conservación de la flora amenazada de Aragón. Zaragoza, Consejo Protecc. Naturaleza de Aragón.

Santa Creu Soler, J. M. (2000): Jomadas de Historia, Economia y Sociedad de El Raspeig. San Vicente de El Raspeig (Alicante), editorial Club Universitario.

Sokal, R.R. y Rohlf, F.J. (1995); Biometry. Nueva York, W.H. Freeman and Company.
Thompson, J.D. (1999): Population differentiation in Mediterranean plants: insights into colonization history and the evolution and conservation of endemic species [Review]. Heredity, 82, p. 3-236.

Thompson, K. (1987): Seed and seed bank. New. Pbytol., 106, p. 23-34.

Tilman, D. (1997): Community invasibility, recruitment limitation, and grassland biodiversity. Ecology, 78, p. 81-92.

Tongway, D.J. y Ludwig, J.A. (1994): Smallscale resource heterogeneity in semi-arid landscapes. Pacific conservation biology, 1 , p. 201-208.

Vilà, M. y Lloret, F. (2000): Seed dynamics of the mast seeding tussock grass Ampelodesmos mauritanica in Mediterranean shrublands. Journal of Ecology, 88 , p. 479-491.

Walker, B.H. (1993): Rangeland ecology: Understanding and managing change. Ambio, 22, p. 80-87.

Waller, D.M. (1993): How does mast-fruiting get started? Tree, 8, p. 122-123.

Wei, Z. y Skarpe, C. (1995): Small-scale species dynamics in semi-arid steppe vegetation in Inner Mongolia. I Veg. Sci. 6, p. 583-592.

Westoby, M., Walker, B.M., y Noy-Meir, I. (1989): Opportunistic management for rangelands not at equilibrim. Journal of Range Management, 42, p. 266-274.

Wiegand, T. y Milton, S.J. (1996): Vegetation change in semiarid communities Simulating probabilities and time scales. Vegetatio, 125, p. 169-183.

Wiegand, T., Milton, S. J., y Wissel, C. (1995): A simulation model for a shrub ecosystem in the semiarid Karoo, South Africa. Ecology, 76, p. 2205-2221. 